\title{
A GENERALIZATION OF A THEOREM OF MAXIMOFF AND APPLICATIONS
}

\author{
BY
}

\section{S. J. AGRONSKY}

\begin{abstract}
Many classes of functions can be characterized in terms of their associated sets. Maximoff gave another type of characterization for the approximately continuous functions. In this paper, we give the conditions under which the two types of characterizations are equivalent. We then show that many classes of functions defined or characterized in terms of their associated sets also admit Maximoff-type characterizations.
\end{abstract}

1. Introduction. For any real-valued function $f$ of a real variable, the associated sets of $f$ are the sets $E^{\alpha}(f)=\{x: f(x)<\alpha\}$ and $E_{\alpha}(f)=\{x: f(x)>\alpha\}$ for real $\alpha$. Many classes of functions can be characterized in terms of their associated sets. For example, a function is continuous if and only if all of its associated sets are open, a function is in Baire Class 1 if and only if all of its associated sets are $F_{\sigma}$ sets, a function is approximately continuous if and only if all of its associated sets are $F_{\sigma}$ sets with the property that every point of an associated set is a point of Lebesgue density of that set. In attempting to obtain an associated set characterization for the class of derivatives Zahorski [5] defined a nested sequence of six classes of functions $\mathfrak{K}_{i}, i=0,1, \ldots, 5$, with $\mathfrak{K}_{0}$ and $\mathfrak{K}_{1}$ both later shown to be the class of Darboux functions in Baire Class $\mathcal{D}_{1}\left(\mathscr{D} \beta_{1}\right)$ and $\mathscr{T}_{5}$ the approximately continuous functions. What accounts for the differences between the classes can be roughly stated as: an associated set for a function in $\mathfrak{M}_{i}$ must be "more dense" near each of its members than an associated set for a function in $\Re_{i-1}$.

Zahorski was able to show that every derivative belongs of $\mathfrak{R}_{3}$ and every bounded derivative belongs to $\mathfrak{R}_{4}$. Since every bounded approximately continuous $\left(\Re_{5}\right)$ function is a derivative, the bounded derivatives were shown to lie between $\Re_{4}$ and bounded $\mathfrak{T}_{5}$. However, Zahorski eventually established that no associated set characterization of the class of bounded derivatives exists. More generally, Bruckner [1] has shown that if $\mathfrak{N}$ is a class of functions characterized in terms of associated sets, then $h \circ f \in \Re$ whenever $f \in \mathfrak{N}$ and $h$ is a homeomorphism. Immediate corollaries to Bruckner's Theorem are that neither the class of bounded derivatives nor the class of derivatives are characterizable in terms of their associated sets. The problem of characterizing these classes is still open.

Received by the editors May 17, 1981 and, in revised form, September 9, 1981.

1980 Mathematics Subject Classification. Primary 26A21; Secondary 26A24.

Key words and phrases. Zahorski's $\mathfrak{K}_{1}$ classes, Darboux-Baire 1 functions. 
In attempting to solve this problem, Maximoff [3] has given a different, rather complicated, type of characterization for the class of approximately continuous functions. Of fundamental importance to Maximoff's Theorem is the Lusin-Menchoff Theorem. We thus have two ways of characterizing classes of functions, the first in terms of associated sets and the second the Maximoff type. The question considered in this paper is: what are the circumstances under which a class of functions which is characterized in one of these two ways admits the other type of characterization? This question is answered by Theorem 1 and as an immediate corollary we get Maximoff's Theorem on approximately continuous functions. The result can be summarized by saying that under certain minimal assumptions and in the presence of an analogue of the Lusin-Menchoff Theorem the two characterizations are equivalent.

In order to show that Theorem 1 applies to classes other than the approximately continuous functions, we must therefore prove various analogues of the LusinMenchoff Theorem. We then see that many classes of functions which are characterized in terms of associated sets (including all of Zahorski's $\mathfrak{K}_{i}$ classes) do admit Maximoff type characterizations.

2. Preliminaries. In what follows, $\mu$ denotes Lebesgue measure, $N$ is the natural numbers and $d(x, I)$ denotes the distance from a point $x$ to an interval $I$.

If $A$ and $E$ are Lebesgue measurable sets and $A \subset E$, then $A \subset{ }_{d} E$ will mean that every point of $A$ is a point of density of $E$. The Lusin-Menchoff Theorem can then be stated as

THEOREM. If $A$ is perfect, $E$ measurable and $A \subset E \subset \subset_{d} E$, then there exists a perfect set $A^{\prime}$ such that $A \subset{ }_{d} A^{\prime} \subset E$.

Analogues of this theorem are obtained by replacing " $d$ " by other suitable properties. To make this precise we have the following

DEFINITION. Let $p$ be a property defined for sets of real numbers (not necessarily all sets) with respect to sets containing them. If $A \subset B$, then we say $A$ is (is not) $p$-contained in $B$, written $A \subset_{p} B\left(A \not \subset_{p} B\right)$ provided $A$ has (does not have) property $p$ with respect to $B$. Then $p$ will be called a strong containment property if $p$ satisfies

(1) If $A \subset \subset_{p} E \subset F$ and $p$ is defined for $A \subset F$, then $A \subset_{p} F$.

(2) If $A \subset E \subset_{p} F$ then $A \subset_{p} F$.

(3) If for each $n \in N, E_{n} \subset_{p} F_{n}$, then $\cup_{n=1}^{\infty} E_{n} \subset \subset_{p} \cup_{n=1}^{\infty} F_{n}$.

Note that ordinary set-containment satisfies (1), (2), and (3). We will be interested in adding various conditions to ordinary containment, thereby obtaining notions of "strong" containment, in such a way that (1), (2), and (3) still hold. Note also that " $d$ " is a strong containment property.

DEFINITION. If $p$ is a strong containment property, we say that $p$ has the Lusin-Menchoff (abbreviated L.M.) property if the direct analogue of the LusinMenchoff Theorem holds for $p$, i.e. whenever $A$ is perfect, $E$ is measurable and $A \subset E \subset_{p} E$, then there exists a perfect set $A^{\prime}$ such that $A \subset_{p} A^{\prime} \subset E$. 
For our purposes it will be useful to consider a weaker version of this condition:

Definition. If $p$ is a strong containment property, we denote by $\mathfrak{R}_{p}$ the set of functions $f$ defined by $f \in \Re_{p}$ if and only if $E^{\alpha}(f) \subset_{p} E^{\alpha}(f) \in \mathscr{F}_{\sigma}$ and $E_{\alpha}(f) \subset_{p}$ $E_{\alpha}(f) \in \mathscr{F}_{\sigma}$ for all real $\alpha$. We then say that $p$ has the weak Lusin-Menchoff property (abbreviated weak L.M.) if whenever $A$ is perfect, $E$ is an associated set for some $f \in \mathfrak{T}_{p}$ and $A \subset E$, then there exists a perfect set $A^{\prime}$ such that $A \subset{ }_{p} A^{\prime} \subset E$.

It is evident that if a property $p$ has the L.M. property, then it also has the weak L.M. property. As we shall see, the converse does not always hold.

In order to state Maximoff's Theorem in a concise way, we make the following

Definition. Let $\left\{y_{1}, y_{2}, \ldots\right\}$ be an enumeration of the rational numbers. If $f$ is any function and $p$ a strong containment property, then a $p$-system for $f$ is a system of perfect sets $\left\{A_{n}^{y_{r}}, A_{y_{r}}^{n}\right\}, r=1,2, \ldots, n=r, r+1, r+2, \ldots$, satisfying

$$
\text { (2) } A_{n}^{y_{r}} \subset_{p} A_{n+1}^{y_{r}} \text {, }
$$

$$
\begin{aligned}
& \bigcup_{n=r}^{\infty} A_{y_{r}}^{n}=E_{y_{r}}(f), \\
& A_{y_{r}}^{n} \subset_{p} A_{y_{r}}^{n+1},
\end{aligned}
$$

and if $y_{s}<y_{t}$ and $n \geqslant \max \{s, t\}$, then

$$
A_{n}^{y_{s}} \subset_{p} A_{n}^{y_{t}} \text { and }
$$

(3') $\quad A_{y_{t}}^{n} \subset_{p} A_{y_{s}}^{n}$

Maximoff's Theorem [3] can now be stated as: A function $f$ is approximately continuous if and only if there exists a $d$-system for $f$.

We will say that a class of functions $\mathfrak{T}$ has a Maximoff-type characterization if there is a strong containment property $p$ such that $\mathfrak{K}^{\prime}=\mathfrak{K}_{p}$. We conclude this section by giving the definitions of Zahorski's $\mathfrak{K}_{i}$ classes [5].

Definition. $f \in \mathscr{R}_{0}$ if and only if all the associated sets of $f$ are $\mathscr{F}_{\sigma}$ sets and whenever $E$ is an associated set of $f, x \in E$ and $\varepsilon>0$ are given, the sets $(x-\varepsilon, x)$ $\cap E$ and $(x, x+\varepsilon) \cap E$ are infinite.

Definition. $f \in \Re_{1}$ if and only if all the associated sets of $f$ are $\mathscr{F}_{\sigma}$ sets and whenever $E$ is an associated set of $f, x \in E$ and $\varepsilon>0$ are given, the sets $(x-\varepsilon, x)$ $\cap E$ and $(x, x+\varepsilon) \cap E$ have cardinality $c$.

Zahorski [5] proved that $\mathfrak{R}_{0}=\mathscr{N}_{1}=\mathscr{D} \beta_{1}$ (the Darboux functions in Baire Class 1).

Definition. $f \in \mathfrak{R}_{2}$ if and only if all the associated sets of $f$ are $\mathscr{F}_{\sigma}$ sets and whenever $E$ is an associated set of $f, x \in E$ and $\varepsilon>0$ are given, the sets $(x-\varepsilon, x)$ $\cap E$ and $(x, x+\varepsilon) \cap E$ have positive measure.

Definition. $f \in \mathscr{T}_{3}$ if and only if all the associated sets of $f$ are $\mathscr{F}_{\sigma}$ sets and whenever $E$ is an associated set of $f$ and $x \in E$ and $k \in N$, there exists $\varepsilon(x, k)>0$ such that $\mu(I \cap E)>0$ whenever $I$ is an interval satisfying

$$
\frac{\mu(I)}{d(x, I)} \geqslant \frac{1}{k} \quad \text { and } \quad \mu(I)+d(x, I) \leqslant \varepsilon(x, k) .
$$

Definition. $f \in \mathfrak{R}_{4}$ if and only if whenever $E$ is an associated set of $f$ there exist a sequence of closed sets $\left\{F_{n}\right\}$ and a sequence of positive numbers $\left\{\eta_{n}\right\}$ such that 
$E=\cup F_{n}$ and whenever $x \in F_{n}$ and $k \in N$, there exists $\varepsilon(x, k)>0$ such that $\mu(I \cap E)>\eta_{n} \mu(I)$ whenever $I$ is an interval satisfying $\mu(I) / d(x, I) \geqslant \frac{1}{k}$ and $\mu(I)+d(x, I) \leqslant \varepsilon(x, k)$.

DEFinition. $f \in \mathfrak{T}_{5}$ if and only if all the associated sets of $f$ are $\mathscr{F}_{\sigma}$ sets and if $E$ is an associated set of $f$, then every point of $E$ is a density point of $E$.

Definition. The class of approximately continuous functions $A$ is defined by $f \in A$ if and only if, for each $x$, there exists a set $D_{x}$ containing $x$ and having $x$ as a density point such that $f / D_{x}$ is continuous at $x .\left(f / D_{x}\right.$ denotes the restriction of $f$ to the domain $D_{x}$.)

Zahorski [5] among others has shown that $A=\Re_{5}$.

3. Main results. We are now ready to prove the theorem giving the conditions under which the associated set and the Maximoff-type characterizations are equivalent.

We begin with a lemma which greatly simplifies the proof of the theorem which follows.

LEMMA 1. Let $p$ be a strong containment property satisfying the weak L.M. condition. Let $f \in \mathfrak{T}_{p}$ and assume additionally that if $E$ is an associated set for $f$ and $x \in E$, then there exists a perfect set $S(x)$ such that $x \in S(x) \subset E$. Then there exists a p-system for $f$. (Note that the additional hypothesis is clearly necessary for the conclusion to be true.)

Proof. We construct the sets $\left\{A_{n}^{y_{r}}\right\}, r \in N, n=r, r+1, \ldots$, the construction of the sets $\left\{A_{y_{r}}^{n}\right\}$ being analogous. Now, since $f \in \mathfrak{M}_{p}$, we have for each $r \in N, E^{y_{r}}(f)$ is an $F_{\sigma}$ set. Since any closed set is a perfect set union a countable set of points, we have $E^{y_{r}}(f)$ expressible as a countable union of perfect sets together with a countable set of points, $\left\{x_{m}\right\}, m \in N$. Now, for each $m \in N, x_{m} \in E^{y_{r}}(f)$ and so by hypothesis there exists a perfect set $S\left(x_{m}\right)$ such that $x_{m} \in S\left(x_{m}\right) \subset E^{y_{r}}(f)$. Hence $E^{y_{r}}(f)$ is expressible as a countable union of perfect sets and we may write $E^{y_{r}}(f)=\cup_{n=r}^{\infty} B_{n}^{y_{r}}, B_{n}^{y_{r}}$ perfect. Note that by (2) since $E^{y_{r}}(f) \subset_{p} E^{y_{r}}(f), B_{n}^{y_{r}} \subset{ }_{p}$ $E^{y_{r}}(f)$ and $\varnothing \subset_{p} E^{y_{r}}(f)$.

It is evident that if we construct a system $\left\{A_{n}^{y_{r}}\right\}_{n \in N, r=n, n+1, \ldots}$ of perfect sets which satisfies for each $n \in N$

$$
\left.A_{n-1}^{y_{j}} \cup B_{n}^{y_{j}} \subset{ }_{p} A_{n}^{y_{j}} \subset E^{y_{j}}(f) \text { for } j=1, \ldots, n \text { (where } A_{n-1}^{y_{n}}=\varnothing\right),
$$

then it will satisfy (1) and (2) in the definition of a p-system. $\left(B_{n}^{y_{j}} \subset A_{n}^{y_{j}} \subset E^{y_{j}}(f)\right.$ implies (1) and $A_{n-1}^{y_{j}} \subset_{p} A_{n}^{y_{j}}$ implies (2).)

It is also evident that condition (3) in the same definition may be restated as, for each $n \in N$,

$$
A_{n}^{y_{\sigma(1)}} \subset_{p} A_{n}^{y_{\sigma(2)}} \subset_{p} \cdots \subset_{p} A_{n}^{y_{\sigma(n)}}
$$

where $\sigma$ is the permutation of $\{1,2, \ldots, n\}$ such that $y_{\sigma(1)}<y_{\sigma(2)}<\cdots<y_{\sigma(n)}$. We construct a system $\left\{A_{n}^{y_{r}}\right\}$ satisfying $(*)$ and $(* *)$ for all $n$ by induction on $n$. For $n=1$, let $A_{1}^{y_{1}}=B_{1}^{y_{1}}$. Then (*) and (**) hold for $n=1$. Assume $A_{n-1}^{y_{1}}, \ldots, A_{n-1}^{y_{n-1}}$ have been defined satisfying $(*)$ and $(* *)$. (Actually only $(*)$ is used.) Let $\sigma$ be the 
permutation of $\{1,2, \ldots, n\}$ such that $y_{\sigma(1)}<y_{\sigma(2)}<\cdots<y_{\sigma(n)}$. We first construct $A_{n}^{y_{\sigma(1)}}$. By (*) (or the convention that $A_{n-1}^{y_{n}}=\varnothing$ if $\sigma(1)=n$ ) we have $B_{n}^{y_{\sigma(1)}} \cup A_{n-1}^{y_{\sigma(1)}}$ is a perfect set $p$-contained in $E^{y_{\sigma(1)}}(f)$, and so since $p$ satisfies the weak L.M. condition, there is a perfect set $A_{n}^{y_{o(1)}}$ such that

$$
B_{n}^{y_{\sigma(1)}} \cup A_{n-1}^{y_{o(1)}} \subset{ }_{p} A_{n}^{y_{\sigma(1)}} \subset E^{y_{o(1)}}(f) \text {. }
$$

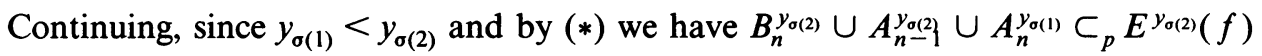
and so again by the weak L.M. property, there exists a perfect set $A_{n}^{y_{\sigma(2)}}$ such that

$$
B_{n}^{y_{\sigma(2)}} \cup A_{n}^{y_{\sigma(2)}} \cup A_{n}^{y_{\sigma(1)}} \subset{ }_{p} A_{n}^{y_{\sigma(2)}} \subset E^{y_{\sigma(2)}}(f) \text {. }
$$

Proceeding in this way we define $A_{n}^{y_{j}}, j=1, \ldots, n$, satisfying (*) and (**). This establishes the induction step and hence the lemma.

THEOREM 1. Let $p$ be a strong containment property satisfying the weak L.M. condition. Further assume that if $E$ is an associated set for some function $f \in \Re_{p}$ and $x \in E$, then there exists a perfect set $S(x)$ such that $x \in S(x) \subset E$. Then $f \in \mathfrak{M}_{p}$ if and only if there exists a p-system for $f$.

Proof. If $f \in \Re_{p}$, we clearly have the hypotheses of Lemma 1 satisfied and so there exists a $p$-system for $f$.

Conversely, assume $f$ is a function such that there exists a $p$-system for $f$, and let $\alpha$ be any real number. We will show that $E^{\alpha}(f)$ is an $F_{\sigma}$ set and $E^{\alpha}(f) \subset_{p} E^{\alpha}(f)$. Observe that $E^{\alpha}(f)=\cup_{y_{r}<\alpha} E^{y_{r}}(f)=\cup_{y_{r}<\alpha} \cup_{n=r}^{\infty} A_{n}^{y_{r}}$.

Since each $A_{n}^{y_{r}}$ is perfect, we have $E^{\alpha}(f)$ an $F_{\sigma}$ set. The condition (3) in the definition of a $p$-system together with condition (3) in the definition of a strong containment property imply $E^{y_{s}}(f) \subset_{p} E^{y_{t}}(f)$ for every $y_{s}<y_{t}$. Then take a sequence $j(r)$ such that $\left\{y_{j(r)}\right\}$ increases to $\alpha$. Then $E^{y_{j(r)}}(f) \subset_{p} E^{y_{j(r+1)}}(f)$ and $\cup_{r=1}^{\infty} E^{y_{j(r)}}(f)=E^{\alpha}(f)$. Thus by (3) in the definition of strong containment, $E^{\alpha}(f) \subset_{p} E^{\alpha}(f)$. Similarly we show $E_{\alpha}(f) \subset_{p} E_{\alpha}(f)$ and the theorem is proved.

It will be useful to note the following:

If $\Re$ is a subclass of $\mathscr{D} \beta_{1}$, and $E$ is an associated set for some $f \in \mathfrak{R}$, and $x \in E$, then there exists a perfect set $S(x)$ such that $x \in S(x) \subset E$. To see this, let $\left\{\varepsilon_{n}\right\}$ be a sequence of positive numbers decreasing to zero such that $x+\varepsilon_{n} \in E$ for all $n$. This is possible since Zahorski's class $\mathscr{N}_{1}$ is known to be $\mathscr{D} \beta_{1}$ [5]. Hence, for each $n$, $\left[x+\varepsilon_{n+1}, x+\varepsilon_{n}\right] \cap E$ has cardinality $c$ and so there exists a perfect set $S_{n} \subset[x+$ $\left.\varepsilon_{n+1}, x+\varepsilon_{n}\right] \cap E$. The set $S=\{x\} \cup \cup_{n=1}^{\infty} S_{n}$ evidently has the desired property.

Since we will only apply Theorem 1 to subclasses of $\mathscr{D} \beta_{1}$, the last hypothesis of the theorem will always be satisfied.

As an immediate application of Theorem 1, we get

COROLlaRY (THEOREM OF MAXIMOFF). $f$ is approximately continuous if and only if there exists a d-system for $f$.

Proof. Clearly " $d$ " is a strong containment property, which by the LusinMenchoff Theorem satisfies the L.M. condition. It is well known (for example, Zahorski [5]) that $\Re_{d}=$ approximately continuous function. Hence all of the hypotheses of Theorem 1 are met and the result follows. 


\section{Applications of Theorem 1.}

Definition. If $A$ and $B$ are sets of real numbers and $A \subset B$, then $A \subset{ }_{p_{0}} B$ will mean that for each $x \in A$ and $\varepsilon>0$, the sets $(x, x+\varepsilon) \cap B$ and $(x-\varepsilon, x) \cap B$ are infinite. It is evident that $\mathfrak{K}_{p_{0}}$ is Zahorski's class $\mathfrak{\Re}_{0}$.

LEMMA 2 (WEAK L.M. PROPERTY FOR $p_{0}$ ). Let $A$ be perfect, $E$ an associated set for some $f \in \Re_{0}$ and $A \subset E$. Then there exists a perfect set $A^{\prime}$ such that $A \subset{ }_{p_{0}} A^{\prime} \subset E$.

Proof. This lemma is implied by Lemma 3 and so the proof is deferred.

THEOREM 2. $f \in \mathfrak{R}_{0}$ if and only if there exists a $p_{0}$-system for $f$.

Proof. Apply Theorem 1.

REMARK. It is easily seen that $p_{0}$ does not enjoy the L.M. property. Let $A$ be any perfect subset of $(0,1)$ and $E$ be the union of $A$ with the rationals. Then $A \subset E \subset{ }_{p_{0}} E$ but if $A^{\prime}$ is a perfect set such that $A \subset A^{\prime} \subset E$, then $A=A^{\prime}$. However $A \not \not_{p_{0}} A$ (since $\checkmark A$ is open), so there is no perfect set $A^{\prime}$ such that $A \subset \subset_{p_{0}} A^{\prime} \subset E$. We note that, therefore, the set $E$ defined above cannot be an associated set for any $f \in \Re_{0}$.

Definition. If $A$ and $B$ are sets of real numbers and $A \subset B$, then $A \subset{ }_{p_{1}} B$ will mean that for each $x \in A$ and $\varepsilon>0$, the sets $(x-\varepsilon, x) \cap B$ and $(x, x+\varepsilon) \cap B$ have cardinality $c$. It is evident that $\mathfrak{R}_{p_{1}}$ is Zahorski's class $\mathfrak{R}_{1}$.

LEMMA 3 (L.M. PROPERTY FOR $p_{1}$ ). Let $A$ be a perfect set, $A \subset E \subset \subset_{p_{1}} E$. Then there exists a perfect set $A^{\prime}$ such that $A \subset_{p_{1}} A^{\prime} \subset E$.

Proof. Let $\left\{\left(a_{n}, b_{n}\right)\right\}, n \in N$, be the sequence of intervals contiguous to $A$.

Choose a sequence of numbers $\varepsilon_{n}^{k}$ by induction to satisfy:

(1) $\varepsilon_{n}^{1}=\frac{1}{2}\left(a_{n}+b_{n}\right)$;

(2) $0<\varepsilon_{n}^{k}<\min \left\{\frac{1}{k}, \varepsilon_{n}^{k-1}\right\}, k=2,3, \ldots$;

(3) $\left(a_{n}+\varepsilon_{n}^{k}, a_{n}+\varepsilon_{n}^{k-1}\right) \cap E$ has cardinality $c$.

Let $B_{n}^{k}$ be a nonempty perfect subset of $\left(a_{n}+\varepsilon_{n}^{k}, a_{n}+\varepsilon_{n}^{k-1}\right) \cap E$. Let $B=\cup_{n=1}^{\infty}$ $\cup_{k=1}^{\infty} B_{n}^{k}$. Similarly choose a sequence $\delta_{n}^{k}$ by induction to satisfy:

(1') $\delta_{n}^{1}=\frac{1}{2}\left(a_{n}+b_{n}\right)$;

(2') $0<\delta_{n}^{k}<\min \left\{\frac{1}{k}, \delta_{n}^{k-1}\right\}, k=2,3, \ldots$;

(3') $\left(b_{n}-\delta_{n}^{k-1}, b_{n}-\delta_{n}^{k}\right) \cap E$ has cardinality $c$.

As above, let $D_{n}^{k}$ be a nonempty perfect subset of $\left(b_{n}-\delta_{n}^{k-1}, b_{n}-\delta_{n}^{k}\right) \cap E$ and let $D=\cup_{n=1}^{\infty} \cup_{k=1}^{\infty} D_{n}^{k}$. Finally, let $A^{\prime}=A \cup B \cup D$. It is clear that $A^{\prime}$ is perfect and that $A \subset A^{\prime} \subset E$. It remains to show $A \subset{ }_{p_{1}} A^{\prime}$. So let $x \in A$ and $\varepsilon>0$ be given. We show that $(x, x+\varepsilon) \cap A^{\prime}$ has cardinality $c$. We have two cases:

(a) $(x, x+\varepsilon) \cap A \neq \varnothing$. Then since $A$ is perfect, $(x, x+\varepsilon) \cap A$ has cardinality $c$ and so a fortiori $(x, x+\varepsilon) \cap A^{\prime}$ has cardinality $c$.

(b) $(x, x+\varepsilon) \cap A=\varnothing$, i.e., $x=a_{n}$ for some $n \in N$. Then since $\varepsilon_{n}^{k}<\frac{1}{k}$, we have that for some $k, \varepsilon_{n}^{k}<\varepsilon$, so $\left(x, x+\varepsilon_{n}^{k}\right) \subset(x, x+\varepsilon)$. But by construction, $(x, x+$ $\left.\varepsilon_{n}^{k}\right) \cap A^{\prime}$ has cardinality $c$ and so $(x, x+\varepsilon) \cap A^{\prime}$ has cardinality $c$.

The fact that $(x-\varepsilon, x) \cap A^{\prime}$ has cardinality $c$ is proved similarly. Hence $A \subset{ }_{p_{1}} A^{\prime}$ and the lemma is proved. 
It is now easy to see how Lemma 3 implies Lemma 2. If $E$ is an associated set for some $f \in \Re_{0}$, then since $\Re_{0}=\Re_{1}$ we know $E \subset \subset_{p_{1}} E$. So if $A$ is perfect and $A \subset E$, by Lemma 3 there exists a perfect set $A^{\prime}$ such that $A \subset \subset_{p_{1}} A^{\prime} \subset E$, so a fortiori $A \subset \subset_{p_{0}} A^{\prime} \subset E$, proving Lemma 2.

THEOREM 3. $f \in \mathfrak{N}_{1}$ if and only if there exists a $p_{1}$-system for $f$.

Proof. Apply Theorem 1.

Definition. If $A$ and $B$ are sets of real numbers and $A \subset B$, then $A \subset \subset_{p_{2}} B$ will mean that for each $x \in A$ and $\varepsilon>0$, the sets $(x-\varepsilon, x) \cap B$ and $(x, x+\varepsilon) \cap B$ have positive measure. It is evident that $\mathscr{R}_{p_{2}}$ is Zahorski's class $\mathfrak{K}_{2}$.

LEMMA 4 (L.M. PROPERTY FOR $p_{2}$ ). Let $A$ be perfect, $A \subset E \subset \subset_{p_{2}} E$. Then there exists a perfect set $A^{\prime}$ such that $A \subset_{p_{2}} A^{\prime} \subset E$.

Proof. The proof is similar to that of Lemma 3. We let $\left\{\left(a_{n}, b_{n}\right)\right\}, n \in N$, be the intervals contiguous to $A$ and we begin by choosing, by induction, sequences of numbers $\varepsilon_{n}^{k}$ and $\delta_{n}^{k}$ to satisfy:

(1) $\varepsilon_{n}^{1}=\delta_{n}^{1} \frac{1}{2}\left(a_{n}+b_{n}\right)$;

(2) $0<\varepsilon_{n}^{k}<\min \left\{\varepsilon_{n}^{k-1}, \frac{1}{k}\right\}, 0<\delta_{n}^{k}<\min \left\{\delta_{n}^{k-1}, \frac{1}{k}\right\}$;

(3) $\left(a_{n}+\varepsilon_{n}^{k}, a_{n}+\varepsilon_{n}^{k-1}\right) \cap E$ has positive measure; $\left(b_{n}-\delta_{n}^{k-1}, b_{n}-\delta_{n}^{k}\right) \cap E$ has positive measure.

Since $E \subset_{p_{2}} E$, we have that

$$
\mu\left(\left(a_{n}+\varepsilon_{n}^{k}, a_{n}+\varepsilon_{n}^{k-1}\right) \cap E\right)>0
$$

and

$$
\mu\left(\left(b_{n}-\delta_{n}^{k-1}, b_{n}-\delta_{n}^{k}\right) \cap E\right)>0,
$$

and so there exist perfect sets

$$
B_{n}^{k} \subset\left(a_{n}+\varepsilon_{n}^{k}, a_{n}+\varepsilon_{n}^{k-1}\right) \cap E
$$

and

$$
D_{n}^{k} \subset\left(b_{n}-\delta_{n}^{k-1}, b_{n}-\delta_{n}^{k}\right) \cap E
$$

such that $\mu\left(B_{n}^{k}\right)>0$ and $\mu\left(D_{n}^{k}\right)>0$. We now let $A^{\prime}=A \cup \cup_{n=1}^{\infty} \cup_{k=1}^{\infty}\left(B_{n}^{k} \cup D_{n}^{k}\right)$. It is clear that $A^{\prime}$ is perfect and that $A \subset A^{\prime} \subset E$. It remains to show $A \subset{ }_{p_{2}} A^{\prime}$. We let $x \in A$ and $\varepsilon>0$ be given. We will show $(x, x+\varepsilon) \cap A^{\prime}$ has positive measure.

We have two cases:

(a) If $(x, x+\varepsilon) \cap A$ has measure zero, then $(x, x+\varepsilon)$ contains some $\left(a_{n}, b_{n}\right)$ in which case $(x, \dot{x}+\varepsilon) \cap A^{\prime}$ has positive measure.

(b) $(x, x+\varepsilon) \cap A=\varnothing$, i.e., $x=a_{n}$ for some $n \in N$. But since $\varepsilon_{n}^{k}<\frac{1}{k}$ we have for some $k, \varepsilon_{n}^{k}<\varepsilon$ and since $\mu\left(\left(x, x+\varepsilon_{n}^{k}\right) \cap A^{\prime}\right)>0$ by construction, we have

$$
\mu\left((x, x+\varepsilon) \cap A^{\prime}\right)>0 .
$$

In exactly the same way we see that $(x-\varepsilon, x) \cap A^{\prime}$ has positive measure. Hence $A \subset_{p_{2}} A^{\prime}$ and the lemma is proved.

THEOREM 4. $f \in \mathfrak{N}_{2}$ if and only if there exists a $p_{2}$-system for $f$. 
Proof. Apply Theorem 1.

Definition. If $A$ and $B$ are measurable sets and $A \subset B$, then $A \subset \subset_{p_{3}} B$ will mean that for each $x \in A$ and $k \in N$, there exists $\varepsilon(x, k)>0$ such that $\mu(I \cap B)>0$ for all intervals $I$ satisfying $\mu(I) / d(x, I) \geqslant \frac{1}{k}$ and $\mu(I)+d(x, I) \leqslant \varepsilon(x, k)$.

Definition. If $A$ and $B$ are measurable sets and $A \subset B$, then $A \subset \subset_{p_{4}} B$ will mean that there exist a sequence $\left\{\eta_{n}\right\}, n \in N$, of positive numbers and a sequence $\left\{F_{n}\right\}$, $n \in N$, of closed sets such that $A=\cup_{n=1}^{\infty} F_{n}$ and for each $x \in F_{n}$ and $k \in N$, there exists $\varepsilon(x, k)>0$ such that $\mu(I \cap B)>\eta_{n} \mu(I)$ for all intervals $I$ for which $\mu(I) / d(x, I) \geqslant \frac{1}{k}$ and $\mu(I)+d(x, I) \leqslant \varepsilon(x, k)$. We note that it is clear that we may assume $\left\{\eta_{n}\right\}$ decreases to zero and $F_{n} \subset F_{n+1}$.

By comparing with the definitions of Zahorski's classes $\mathfrak{R}_{3}$ and $\mathfrak{R}_{4}$, one easily sees that $\mathfrak{K}_{3}=\mathfrak{K}_{p_{3}}$ and $\mathfrak{M}_{4}=\mathfrak{K}_{p_{4}}$.

The proofs that $p_{3}$ and $p_{4}$ have the L.M. property are rather long. We will give a proof for $p_{4}$ and then indicate how it can be adapted to give a proof for $p_{3}$.

LEMMA 5 (L.M. PROPERTY FOR $p_{4}$ ). If $A$ is a perfect set and $A \subset E \subset_{p_{4}} E$, then there exists a perfect set $A^{\prime}$ such that $A \subset{ }_{p_{4}} A^{\prime} \subset E$.

Proof. To prove the lemma we must construct a perfect set $A^{\prime} \subset E$ such that there exist a sequence $\left\{\delta_{n}\right\}, n \in N$, of positive numbers and a sequence $\left\{G_{n}\right\}, n \in N$, of closed sets such that $A=\cup_{n \in N} G_{n}$ and if $x \in G_{n}$ then for each $k \in N$ there exists $\lambda(x, k)>0$ such that $\mu\left(I \cap A^{\prime}\right)>\delta_{n} \mu(I)$ for all intervals $I$ for which

$$
\frac{\mu(I)}{d(x, I)} \geqslant \frac{1}{k} \text { and } \mu(I)+d(x, I) \leqslant \lambda(x, k) \text {. }
$$

Now, $A \subset E \subset \subset_{p_{4}} E$ means, by the above definition, that there exist $\left\{F_{n}\right\}$ closed sets and $\left\{\eta_{n}\right\}$ positive numbers such that $E=\cup_{n \in N} F_{n}$ and for each $x \in F_{n}$ and $k \in N$, there exists $\varepsilon(x, k)>0$ such that $\mu(I \cap E)>\eta_{n} \mu(I)$ for all intervals $I$ for which $\mu(I)+d(x, I) \leqslant \varepsilon(x, k)$ and $\mu(I) / d(x, I) \geqslant \frac{1}{k}$. We will let $\delta_{n}=\eta_{n} / 24$ and $G_{n}=A$ $\cap F_{n}$.

Before beginning the construction of $A^{\prime}$, it is useful to give an outline. The construction of $A^{\prime}$ will be done in two parts. Let $\left\{\left(a_{s}, b_{s}\right)\right\}, s \in N$, be the intervals contiguous to $A$. In Part 1 we construct, for each $s \in N$, a perfect set $B_{r}^{s} \subset\left[a_{s}, b_{s}\right] \cap$ $E$ having the property that if $a_{s} \in F_{n} \backslash F_{n-1}$, then for each $k \in N$ there exists $\lambda_{r}\left(a_{s}, k\right)>0$ such that $\mu\left(I \cap B_{r}^{s}\right)>\eta_{n} \mu(I) / 24$ for all intervals $I$ lying to the right of $a_{s}$ for which $\mu(I)+d\left(a_{s}, I\right) \leqslant \lambda_{r}\left(a_{s}, k\right)$ and $\mu(I) / d\left(a_{s}, I\right) \geqslant \frac{1}{k}$. We will then let $B_{r}=\cup_{s=1}^{\infty} B_{r}^{s}$ and we will clearly have that $A \cup B_{r}$ is a perfect subset of $E$. After constructing $B_{r}$ it is then evident that there exists a set $B_{l}$ such that $A \cup B_{l}$ is perfect and for each $s \in N$, if $b_{s} \in F_{m} \backslash F_{m-1}$, then for each $k \in N$ there exists $\lambda_{1}\left(b_{s}, k\right)>0$ such that $\mu\left(I \cap B_{l}\right)>\eta_{m} \mu(I) / 24$ for all intervals $I$ lying to the left of $b_{s}$ for which $\mu(I) / d\left(b_{s}, I\right) \geqslant \frac{1}{k}$ and $\mu(I)+d\left(b_{s}, I\right) \geqslant \lambda_{1}\left(b_{s}, k\right)$. We then let $B=B_{r} \cup B_{l}$ and we have $A \cup B$ is a perfect subset of $E$. In Part 2 we construct a set $D \subset E$ in such a way that $A \cup D$ is perfect and the set $A^{\prime}=A \cup B \cup D$ has the desired property, i.e., $A \subset{ }_{p_{4}} A^{\prime}$. 
Part 1. Fix $\left(a_{s}, b_{s}\right)$ and we construct $B_{r}^{s}$. Suppose $a_{s} \in F_{n} \backslash F_{n-1}$. Choose inductively (on $k)$ a sequence of positive numbers $\lambda_{r}\left(a_{s}, k\right)$ to satisfy:

$$
\begin{gathered}
\lambda_{r}\left(a_{s}, 1\right)=\min \left\{\frac{1}{2}\left(b_{s}-a_{s}\right), \varepsilon\left(a_{s}, 2\right)\right\} ; \\
\lambda_{r}\left(a_{s}, k\right) \leqslant \varepsilon\left(a_{s}, 2 k\right) . \\
\lambda_{r}\left(a_{s}, k+1\right)=\left(\frac{2 k}{2 k+1}\right)^{i_{k}} \lambda_{r}\left(a_{s}, k\right) \text { for some } i_{k} \in N .
\end{gathered}
$$

Now, for each $k$, partition the interval $\left[a_{s}+\lambda_{r}\left(a_{s}, k+1\right), a_{s}+\lambda_{r}\left(a_{s}, k\right)\right]$ into nonoverlapping subintervals $I_{s}^{k}[j]$ for $j=1,2, \ldots, i_{k}$ in such a way that

$$
\frac{\mu\left(I_{s}^{k}[j]\right)}{d\left(a_{s}, I_{s}^{k}[j]\right)}=\frac{1}{2 k}
$$

for each $j=1,2, \ldots, i_{k}$. (This is possible by (3) above.) Then, for each $k \in N$ and $j$ between 1 and $i_{k}$, we have that

$$
\mu\left(I_{s}^{k}[j]\right)+d\left(a_{s}, I_{s}^{k}[j]\right) \leqslant \lambda_{r}\left(a_{s}, k\right) \leqslant \varepsilon\left(a_{s}, 2 k\right),
$$

and so

$$
\mu\left(I_{s}^{k}[j] \cap E\right)>\eta_{n} \mu\left(I_{s}^{k}[j]\right) .
$$

Hence there exists a perfect set $B_{r}^{s}[k, j] \subset I_{s}^{k}[j] \cap E$ such that $\mu\left(I_{s}^{k}[j] \cap B_{r}^{s}[k, j]\right)$ $>\eta_{n} \mu\left(I_{s}^{k}[j]\right)$. Let $B_{r}^{s}=\cup_{k=1}^{\infty} \cup_{j=1}^{i_{k}} B_{r}^{s}[k, j]$. Then it is clear that $A \cup B_{r}^{s}$ is a perfect subset of $E$ and we claim that $B_{r}^{s}$ has the property described in the outline. So we let $k \in N$ and $I=[x, y]$ be an interval lying to the right of $a_{s}$ such that $\mu(I)+d\left(a_{s}, I\right) \leqslant \lambda_{r}\left(a_{s}, k\right)$ and $\mu(I) / d\left(a_{s}, I\right) \geqslant \frac{1}{k}$. Then clearly, for some $m \geqslant k$ and some $j$ between 1 and $i_{m}$, there is an interval $I_{s}^{m}[j] \subset I$. So we write $I=I_{1} \cup I_{2}$ $\cup I_{3}$ where $I_{2}$ is the union of all the intervals $I_{s}^{t}[j]$ which are completely contained in $I$ and $I_{1}=I \cap I_{s}^{t_{1}}\left[j_{1}\right]$ and $I_{3}=I_{s}^{t_{2}}\left[j_{2}\right]$ where $I_{s}^{t_{1}}\left[j_{1}\right]$ and $I_{s}^{t_{2}}\left[j_{2}\right]$ contain $x$ and $y$ in their respective interiors. If $x(y)$ is already in $I_{2}$, we take $I_{1}\left(I_{3}\right)$ to be empty. We have seen that $I_{2} \neq \varnothing$ and so it is clear that $\mu\left(I_{2}\right)>\mu\left(I_{1}\right)$ and $2 \mu\left(I_{2}\right)>\mu\left(I_{2}\right)$ and so $4 \mu\left(I_{2}\right)>\mu(I)$. Hence

$$
\frac{\mu\left(I \cap B_{r}^{s}\right)}{\mu(I)} \geqslant \frac{\mu\left(I_{2} \cap B_{r}^{s}\right)}{\mu(I)}>\frac{\mu\left(I_{2} \cap B_{r}^{s}\right)}{4 \mu\left(I_{2}\right)}>\frac{\eta_{n}}{4}>\frac{\eta_{n}}{24} .
$$

Thus $B=\cup_{s=1}^{\infty} B_{r}^{s}$ has the desired property. Analogously, we construct the set $B_{l}$ as described in the outline and, letting $B=B_{r} \cup B_{l}$, we complete Part 1 of the construction of $A^{\prime}$.

Part 2. Fix $s \in N$ and we construct $D_{1}^{s}=D_{1} \cap\left[a_{s}, b_{s}\right]$. We first define inductively the decreasing sequence $\alpha_{m}^{s}, m=0,1,2, \ldots$, by $\alpha_{0}^{s}=b_{s}$ and $\alpha_{m}^{s}$ is defined so that if $J_{m}^{s}=\left[\alpha_{m}^{s}, \alpha_{m-1}^{s}\right]$, then $\mu\left(J_{m}^{s}\right) / d\left(a_{s}, J_{m}^{s}\right)=1 / 2 s$. Now let $D_{1}^{s}$ be a perfect subset of $\left[a_{s}, b_{s}\right] \cap E$ such that $\mu\left(D_{1}^{s} \cap J_{m}^{s}\right) \geqslant\left(1-\eta_{s} / 6\right) \mu\left(E \cap J_{m}^{s}\right)$ for all $m \in N$. (Let $D_{1}^{s} \cap$ $J_{m}^{s}=\varnothing$ if $\mu\left(E \cap J_{m}^{s}\right)=0$.) It is clear that if $I$ is an interval and $I \subset\left[a_{s}, b_{s}\right]$ and $\mu(I) / d\left(a_{s}, I\right) \geqslant \frac{1}{s}$, then $I$ contains at least one of the intervals $J_{m}^{s}$ for some $m$. 
Claim 1. If $I$ is a closed interval and $I \subset\left[a_{s}, b_{s}\right]$ and $I$ contains at least one of the intervals $J_{m}^{s}$ for some $m \in N$, and $\mu(I \cap E)>\eta \mu(I)$ for some $\eta \geqslant \eta_{s}$, then

$$
\mu\left(I \cap D_{1}^{s}\right)>\eta \mu(I) / 6 .
$$

Proof of Claim 1. We write $I$ as $I=I_{1} \cup I_{2} \cup I_{3}$ where $I_{2}$ is the union of all intervals $J_{m}^{s}$ contained in $I$ and $I_{1}=J_{m_{1}}^{s} \cap I$ and $I_{3}=J_{m_{2}}^{s} \cap I$ where $J_{m_{1}}^{s}$ and $J_{m_{2}}^{s}$ contain the left and right endpoints of $I$ respectively. (If the left (right) endpoint of $I$ is in $I_{2}$, then $I_{1}\left(I_{3}\right)$ is empty.) Now, since $s \geqslant 1$ and $\mu\left(J_{m}^{s}\right) / d\left(a_{s}, J_{m}^{s}\right)=1 / 2 s$ and $I_{2} \neq \varnothing$, it is clear that $\mu\left(J_{m_{1}}^{s}\right)<\mu\left(I_{2}\right)$ and $\mu\left(J_{m_{2}}^{s}\right)<2 \mu\left(I_{2}\right)$. Hence

$$
\mu(I)>\frac{1}{3} \mu\left(J_{m_{1}}^{s} \cup J_{m_{2}}^{s}\right)
$$

and so $\mu(I \cap E)>\frac{\eta}{3} \mu\left(J_{m_{1}}^{s} \cup J_{m_{2}}^{s}\right)$. We have two cases:

Case 1. $\mu\left(\left(I_{1} \cup I_{3}\right) \cap E\right) \geqslant \frac{3}{5} \mu(I \cap E)$; in which case

$$
\mu\left(\left(I_{1} \cup I_{3}\right) \cap E\right)>\frac{3}{5} \frac{\eta}{3} \mu\left(J_{m_{1}}^{s} \cup J_{m_{2}}^{s}\right)=\frac{\eta}{5} \mu\left(J_{m_{1}}^{s} \cup J_{m_{2}}^{s}\right) .
$$

Then

$$
\begin{aligned}
\mu\left(\left(I_{1} \cup I_{3}\right) \cap D_{1}^{s}\right) & >\mu\left(\left(I_{1} \cup I_{3}\right) \cap E\right)-\frac{\eta_{s}}{6} \mu\left(J_{m_{1}}^{s} \cup J_{m_{2}}^{s}\right) \\
& \geqslant \mu\left(\left(I_{1} \cup I_{3}\right) \cap E\right)-\frac{\eta}{6} \mu\left(J_{m_{1}}^{s} \cup J_{m_{2}}^{s}\right)
\end{aligned}
$$

since $\eta \geqslant \eta_{s}$. So

$$
\frac{\mu\left[\left(I_{1} \cup I_{3}\right) \cap D_{1}^{s}\right]}{\mu\left[\left(I_{1} \cup I_{3}\right) \cap E\right]}>1-\frac{(\eta / 6) \mu\left(J_{m_{1}}^{s} \cup J_{m_{2}}^{s}\right)}{\mu\left[\left(I_{1} \cup I_{3}\right) \cap E\right]} .
$$

But, by the hypothesis of Case $1, \mu\left[\left(I_{1} \cup I_{3}\right) \cap E\right]>(\eta / 5) \mu\left(J_{m_{1}}^{s} \cup J_{m_{2}}^{s}\right)$, so we get

$$
\frac{\mu\left[\left(I_{1} \cup I_{3}\right) \cap D_{1}^{s}\right]}{\mu\left[\left(I_{1} \cup I_{3}\right) \cap E\right]}>1-\frac{(\eta / 6) \mu\left(J_{m_{1}}^{s} \cup J_{m_{2}}^{s}\right)}{(\eta / 5) \mu\left(J_{m_{1}}^{s} \cup J_{m_{2}}^{s}\right)}=\frac{1}{6} \text {. }
$$

So $\mu\left[\left(I_{1} \cup I_{3}\right) \cap D_{1}^{s}\right]>\frac{1}{6} \mu\left[\left(I_{1} \cup I_{3}\right) \cap E\right]$ and since by construction $\mu\left(I_{2} \cap D_{1}^{s}\right)$ $\geqslant \frac{5}{6}\left(I_{2} \cap E\right)$, we get

$$
\mu\left(I \cap D_{1}^{s}\right)>\frac{1}{6} \mu(I \cap E)>\frac{n}{6} \mu(I),
$$

proving the claim for Case 1 .

Case 2. $\mu\left(I_{2} \cap E\right)>\frac{2}{5} \mu(I \cap E)$. Since

$$
\mu\left(I_{2} \cap D_{1}^{s}\right)>\left(1-\eta_{s} / 6\right) \mu\left(I_{2} \cap E\right)>\frac{5}{6} \mu\left(I_{2} \cap E\right)
$$

we have

$$
\mu\left(I \cap D_{1}^{s}\right)>\mu\left(I_{2} \cap D_{1}^{s}\right)>\frac{5}{6} \frac{2}{5} \mu(I \cap E)=\frac{1}{3} \mu(I \cap E)>\frac{\eta}{3} \mu(I)>\frac{\eta}{6} \mu(I) .
$$

This establishes the claim for Case 2 and hence finishes the proof of Claim 1.

We now define analogously the perfect set $D_{2}^{s}$ by first defining the increasing sequence $\beta_{m}^{s}, m=0,1,2, \ldots$, where $\beta_{0}^{s}=a_{s}$ and $\beta_{m}^{s}$ is defined so that

$$
\frac{\mu\left(\left[\beta_{m-1}^{s}, \beta_{m}^{s}\right]\right)}{d\left(b_{s},\left[\beta_{m-1}^{s}, \beta_{m}^{s}\right]\right)}=\frac{1}{2 s} \text {. }
$$


We then let $D_{2}^{s}$ be a perfect subset of $\left[a_{s}, b_{s}\right] \cap E$ such that

$$
\mu\left(D_{2}^{s} \cap\left[\beta_{m-1}^{s}, \beta_{m}^{s}\right]\right) \geqslant\left(1-\eta_{s} / 6\right) \mu\left(E \cap\left[\beta_{m-1}^{s}, \beta_{m}^{s}\right]\right) .
$$

Exactly as for $D_{1}^{s}$, we see that if $I \subset\left[a_{s}, b_{s}\right]$ and $\mu(I) / d\left(b_{s}, I\right) \geqslant \frac{1}{s}$, then $I$ contains at least one of the intervals $\left[\beta_{m-1}^{s}, \beta_{m}^{s}\right]$ for some $m \in N$. Also, analogous to Claim 1 we get

Claim $1^{\prime}$. If $I$ is a closed interval, $I \subset\left[a_{s}, b_{s}\right]$ and $I$ contains at least one $\left[\beta_{m-1}, \beta_{m}\right]$ and $\mu(I \cap E)>\eta \mu(I)$ for some $\eta \geqslant \eta_{s}$, then $\mu\left(I \cap D_{2}^{s}\right)>\eta \mu(I) / 6$.

We now let $D_{1}=\cup_{s=1}^{\infty} D_{1}^{s}, D_{2}=\cup_{s=1}^{\infty} D_{2}^{s}, D=D_{1} \cup D_{2}$ and finally, $A^{\prime}=A \cup B$ $\cup D$. It is clear that $A^{\prime}$ is perfect and that $A \subset A^{\prime} \subset E$. It remains to show $A \subset{ }_{p_{4}} A^{\prime}$. We let $x \in A \cap F_{n}$ (and assume $x \notin F_{n-1}$ ) and $k \in N$. We first show that there exists $\lambda_{r}(x, k)>0$ such that $\mu\left(I \cap A^{\prime}\right)>\eta_{n} \mu(I) / 24$ for all intervals $I$ lying to the right of $x$ for which $\mu(I) / d(x, I) \geqslant \frac{1}{k}$ and $\mu(I)+d(x, I) \leqslant \lambda_{r}(x, k)$. We have three possibilities:

(1) $x=a_{s}$ for some $s \in N$. Then we have seen in Part 1 that there exists $\lambda_{r}\left(a_{s}, k\right)>0$ such that $\mu\left(I \cap B_{1}^{s}\right)>\eta_{n} \mu(I) / 24$ for all intervals $I$ lying to the right of $a_{s}$ for which $\mu(I) / d\left(a_{s}, I\right) \geqslant \frac{1}{k}$ and $\mu(I)+d\left(a_{s}, I\right) \leqslant \lambda_{r}\left(a_{s}, k\right)$. Hence

$$
\mu\left(I \cap A^{\prime}\right) \geqslant \mu\left(I \cap B_{1}^{s}\right)>\eta_{n} \mu(I) / 24
$$

for all such intervals $I$.

(2) There exists $\varepsilon>0$ such that $(x, x+\varepsilon) \subset A$. In this case the condition is clearly met by taking $\lambda_{r}(x, k) \leqslant \varepsilon$.

(3) $x$ is a limit point from the right of a subsequence of $\left\{\left(a_{s}, b_{s}\right)\right\}$. In this case, we choose $\lambda_{r}(x, k)$ to be positive number so small that

(a) $\left(x, x+\lambda_{r}(x, k)\right) \cap\left(a_{s}, b_{s}\right)=\varnothing$ for all $s \leqslant k$.

(b) $\lambda_{r}(x, k) \leqslant \varepsilon(x, k)$.

(c) $\left(x, x+\lambda_{r}(x, k)\right) \cap\left(a_{s}, b_{s}\right) \neq \varnothing$ only if $\frac{1}{4} \eta_{n}>\eta_{s}$.

We then let $I$ be a closed interval lying to the right of $x$ for which $\mu(I) / d(x, I) \geqslant \frac{1}{k}$ and $\mu(I)+d(x, I) \leqslant \lambda_{r}(x, k)$. We need to show $\mu\left(I \cap A^{\prime}\right)>\eta_{n} \mu(I) / 24$. Let $I=$ $[\alpha, \beta]$ and write

$$
I \cap E=(I \cap A) \cup(I \cap E \cap \backslash A)
$$

We first observe that if $\mu(I \cap A)>\frac{1}{6} \mu(I \cap E)$, then

$$
\mu\left(I \cap A^{\prime}\right) \geqslant \mu(I \cap A)>\frac{1}{6} \mu(I \cap E)>\frac{\eta_{n}}{6} \mu(I)>\frac{\eta_{n}}{24} \mu(I)
$$

and so we are done. Hence we assume

$$
\mu(I \cap E \cap \backslash A) \geqslant \frac{5}{6} \mu(I \cap E)>\frac{5}{6} \eta_{n} \mu(I) .
$$

We write $I \cap E \cap \backslash A=\left(\cup_{s \in N^{\prime}}\left(a_{s}, b_{s}\right) \cap E\right) \cup\left(\left(I_{1} \cup I_{2}\right) \cap E\right)$ where $s \in N^{\prime}$ if and only if $\left(a_{s}, b_{s}\right) \subset I$ and $I_{1}=\left[\alpha, b_{s_{1}}\right]$ and $I_{2}=\left[a_{s_{2}}, \beta\right]$. We have two cases: 
Case 1. $\mu\left(\cup_{s \in N^{\prime}}\left(a_{s}, b_{s}\right) \cap E\right)>\frac{2}{5} \mu(I \cap E \cap \backslash A) \geqslant \frac{2}{5} \cdot \frac{5}{6} \mu(I \cap E)>\eta_{n} \mu(I) / 3$. Then

$$
\begin{aligned}
\mu\left(I \cap A^{\prime}\right) & \geqslant \mu\left(\bigcup_{s \in N^{\prime}}\left(a_{s}, b_{s}\right) \cap D_{1}\right)=\sum_{s \in N^{\prime}} \mu\left[\left(a_{s}, b_{s}\right) \cap D_{1}^{s}\right] \\
& >\sum_{s \in N^{\prime}}\left[1-\frac{\eta_{s}}{6}\right]\left[\mu\left(a_{s}, b_{s}\right) \cap E\right]>\frac{5}{6} \sum_{s \in N^{\prime}} \mu\left(a_{s}, b_{s}\right) \cap E \\
& =\frac{5}{6} \mu\left(\bigcup_{s \in N^{\prime}}\left(a_{s}, b_{s}\right) \cap E\right)>\frac{5}{6} \frac{\eta_{n}}{3} \mu(I)>\frac{\eta_{n}}{24} \mu(I) .
\end{aligned}
$$

Case 2.

$$
\mu\left[\left(I_{1} \cup I_{2}\right) \cap E\right] \geqslant \frac{3}{5} \mu(I \cap E \cap \backslash A)>\frac{3}{5} \frac{5}{6} \eta_{n} \mu(I)=\frac{1}{2} \eta_{n} \mu(I) .
$$

Clearly $\mu\left(I_{1} \cap E\right) \geqslant \frac{1}{2} \mu\left[\left(I_{1} \cup I_{2}\right) \cap E\right]$ or $\mu\left(I_{2} \cap E\right) \geqslant \frac{1}{2} \mu\left[\left(I_{1} \cup I_{2}\right) \cap E\right]$ and so by the obvious symmetry, we lose no generality in assuming

$$
\mu\left(I_{1} \cap E\right) \geqslant \frac{1}{2} \mu\left[\left(I_{1} \cup I_{2}\right) \cap E\right]>\frac{1}{4} \eta_{n} \mu(I) .
$$

We write

$$
\frac{1}{4} \eta_{n} \mu(I)=\frac{1}{4} \eta_{n} \frac{\mu(I)}{\mu\left(I_{1}\right)} \cdot \mu\left(I_{1}\right) .
$$

Now, $\mu(I) \geqslant \mu\left(I_{1}\right)$ and $\frac{1}{4} \eta_{n}>\eta_{s_{1}}$ by (c) and so $\frac{1}{4} \eta_{n} \mu(I) / \mu\left(I_{1}\right)>\eta_{s_{1}}$. Also, since $I_{1}=\left[\alpha, b_{s_{1}}\right], I_{1}$ clearly contains intervals of the form $\left(\beta_{m}^{s_{1}}, \beta_{m+1}^{s_{1}}\right)$. Therefore, by Claim $1^{\prime}$ with $\frac{1}{4} \eta_{n} \mu(I) / \mu\left(I_{1}\right)$ playing the role of $\eta$, we get

$$
\mu\left(I \cap A^{\prime}\right) \geqslant \mu\left(I \cap D_{2}^{s}\right)>\frac{1}{6} \frac{1}{4} \eta_{n} \frac{\mu(I)}{\mu\left(I_{1}\right)} \cdot \mu\left(I_{1}\right)=\frac{\eta_{n}}{24} \mu(I) .
$$

We have thus exhausted all possibilities.

In the same way, we see that there exists $\lambda_{1}(x, k)>0$ such that $\mu\left(I \cap A^{\prime}\right)>$ $\eta_{n} \mu(I) / 24$ for all intervals $I$ lying to the left of $x$ for which $\mu(I) / d(x, I) \geqslant \frac{1}{k}$ and $\mu(I)+d(x, I) \leqslant \lambda_{1}(x, k)$. Hence by letting $\lambda(x, k)=\min \left\{\lambda_{r}(x, k), \lambda_{1}(x, k)\right\}$ we see that $A \subset_{p_{4}} A^{\prime}$ and so the lemma is proved.

THEOREM 5. $f \in \mathfrak{R}_{4}$ if and only if there exists a $p_{4}$-system for $f$.

Proof. Apply Theorem 1.

In order to prove that $p_{3}$ has the L.M. property, we may proceed in the same way as in Lemma 5. We are given $A \subset E \subset \subset_{p_{3}} E$ where $A$ is perfect and we wish to construct a perfect set $A^{\prime}$ so that $A \subset{ }_{p_{3}} A^{\prime} \subset E$. Again the construction can be broken into two parts and we need only require $\mu(G \cap E)>0$ for $G$ any of the (perfect) sets $B_{r}^{s}[k, j], B_{l}^{s}[k, j], D_{1}^{s}, D_{2}^{s}$ for $k, j, s \in N$. The result follows easily. Hence, by applying Theorem 1 to $p_{3}$, we see that Zahorski's class $\mathfrak{R}_{3}$ also has a Maximoff-type characterization.

In conclusion we remark that, by defining suitable strong containment properties, and showing they have the weak L.M. property, one can show other related classes of functions also admit Maximoff-type characterizations. Among those are Weil's 
class $Z$ [4] and a class $\Re_{p_{*}}$ defined (but not labeled, the designation $\mathscr{T}_{p_{*}}$ is the author's) by Zahorski [5, p. 52]. In the same paper, Zahorski asked if $\mathfrak{K}_{p_{*}}=\mathfrak{K}_{4}$ and Lipiński [2] eventually answered in the negative showing $\mathfrak{K}_{p_{*}} \subsetneq \mathfrak{K}_{4}$.

\section{REFERENCES}

1. A. M. Bruckner, On characterizing classes of functions in terms of associated sets, Canad. Math. Bull. 10 (2) (1967), 227-231.

2. J. Lipiński, Sur certains problemes de Choquet et de Zahorski concernant les fonctions dériveés, Fund. Math. 44 (1957), 94-102.

3. I. Maximoff, On approximately continuous functions, Bull. Amer. Math. Soc. (1939), 264-268.

4. C. Weil, A property for certain derivatives, Indiana Univ. Math. J. 23 (1973-74), 527-536.

5. Z. Zahorski, Sur la première dérivée, Trans. Amer. Math. Soc. 69 (1950), 1-54.

Department of Mathematics, University of California, Davis, California 95616

Current address: Department of Mathematics, California Polytechnic State University, San Luis Obispo, California 93407 